\title{
A SEQUENTIAL RIESZ-LIKE CRITERION FOR THE RIEMANN HYPOTHESIS
}

LUIS BÁEZ-DUARTE

Received 7 June 2005 and in revised form 18 August 2005

Let $c_{k}:=\sum_{j=0}^{k}(-1)^{j}\left(\begin{array}{c}k \\ j\end{array}\right)(1 / \zeta(2 j+2))$. We prove that the Riemann hypothesis is equivalent to $c_{k} \ll k^{-3 / 4+\epsilon}$ for all $\epsilon>0$; furthermore, we prove that $c_{k} \ll k^{-3 / 4}$ implies that the zeros of $\zeta(s)$ are simple. This is closely related to M. Riesz's criterion which states that the Riemann hypothesis is equivalent to $\sum_{k=1}^{\infty}\left((-1)^{k+1} x^{k} /(k-1) ! \zeta(2 k)\right) \ll x^{1 / 4+\epsilon}$ as $x \rightarrow+\infty$, for all $\epsilon>0$.

\section{Introduction and preliminaries}

The main theorem of this note is the following theorem.

Theorem 1.1. Let

$$
c_{k}:=\sum_{j=0}^{k}(-1)^{j}\left(\begin{array}{l}
k \\
j
\end{array}\right) \frac{1}{\zeta(2 j+2)} ;
$$

then the Riemann hypothesis is true if and only if

$$
c_{k} \ll k^{-3 / 4+\epsilon}, \quad \forall \epsilon>0 .
$$

Furthermore, if $c_{k} \ll k^{-3 / 4}$, then the zeros of $\zeta(s)$ are simple.

The proof of this theorem is given in Section 3.

Remark 1.2. It will be seen below that unconditionally

$$
c_{k} \ll k^{-1 / 2}
$$

and, on the other hand, that

$$
c_{k} k k^{-3 / 4-\delta} \quad(\forall \delta>0) .
$$


Remark 1.3. It is quite obvious how one can trivially modify the proof of the theorem to obtain a more general result.

Theorem 1.4. A necessary and sufficient condition for $\zeta(s) \neq 0$ in the half-plane $\mathfrak{R}(s)>$ $2(1-\alpha)$ is

$$
c_{k} \ll k^{-\alpha+\epsilon} \quad(\forall \epsilon>0) .
$$

However, we will eschew such gratuitous generalizing at this stage.

Necessary and sufficient conditions for the Riemann hypothesis depending only on values of $\zeta(s)$ at positive integers have been known for a long time, for example those of Riesz [7] and Hardy and Littlewood [3]. Riesz's criterion, for example, states that the Riemann hypothesis is true if and only if

$$
\sum_{k=1}^{\infty} \frac{(-1)^{k+1} x^{k}}{(k-1) ! \zeta(2 k)}=O\left(x^{1 / 4+\epsilon}\right) \quad(x \longrightarrow+\infty) .
$$

We believe our condition is new and it is definitely simpler, as it only involves finite rational combinations of the values $\zeta(2 h)$, and seems well posed for numerical calculations. This work however did not originate as an attempt to simplify Riesz's criterion. It arose rather as a consequence of our note [1] on Maslanka's expression of the Riemann zeta function $[4,5]$ in the form

$$
(s-1) \zeta(s)=\sum_{k=0}^{\infty} A_{k} P_{k}\left(\frac{s}{2}\right)
$$

where

$$
A_{k}=\sum_{j=0}^{k}(-1)^{j}\left(\begin{array}{l}
k \\
j
\end{array}\right)(2 j+1) \zeta(2 j+2),
$$

and the $P_{k}(s)$ are the Pochhammer polynomials

$$
P_{k}(s):=\prod_{r=1}^{k}\left(1-\frac{s}{r}\right)
$$

which will appear prominently in the proof of Theorem 1.1. The necessary elementary facts about these polynomials are proved in Section 2.

In Section 4, we prove an unconditional exact formula for the coefficients $c_{k}$, stated in the following theorem, where we denote

$$
R_{k}(\omega):=\operatorname{Res}\left(\frac{1}{\zeta(s) P_{k}(s / 2)} ; s=\omega\right)
$$




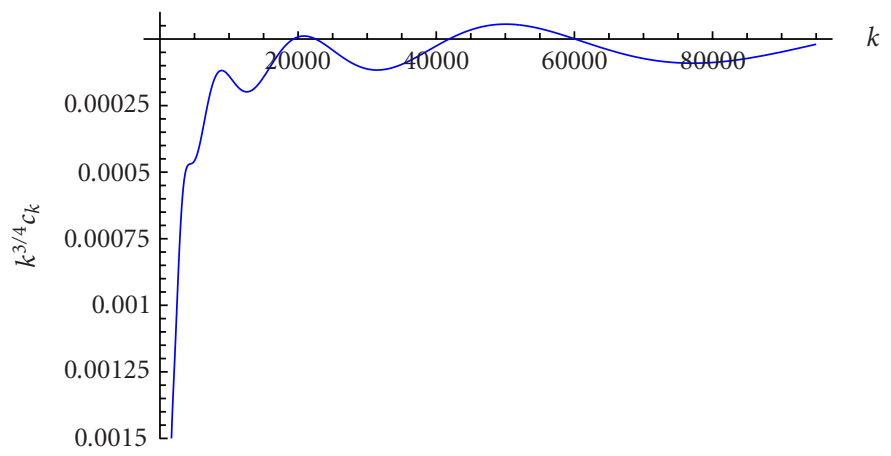

Figure 1.1

Theorem 1.5 (explicit formula). For sufficiently large $k$,

$$
-2 k c_{k-1}=\lim _{\nu \rightarrow \infty} \sum_{|\mathfrak{J} \rho|<T_{\nu}} R_{k}(\rho)+o(1)
$$

where $\left\{T_{\nu}\right\}_{\nu=0}^{\infty}$ is a certain sequence satisfying $\nu<T_{\nu}<\nu+1$, and the $\rho$ denote complex zeta zeros. If simple zeros are assumed, then the above series becomes

$$
-2 k c_{k-1}=\lim _{\nu \rightarrow \infty} \sum_{|\mathfrak{I} \rho|<T_{\nu}} \frac{1}{\zeta^{\prime}(\rho) P_{k}(\rho / 2)}+o(1) .
$$

Remark 1.6. The $o(1)$ can be written explicitly in terms of the trivial zeros $-2,-4, \ldots$ of $\zeta(s)$. This representation is the initial step to prove this conjecture that the condition $c_{k} \ll k^{-3 / 4}$ is both a necessary and sufficient condition for the Riemann hypothesis with simple zeros. The sufficiency is indeed true as will be seen below.

Some extended numerical computations of the $c_{k}$ were kindly carried out for the author by K. Maslanka (personal communication). They seem to indicate good agreement with even $c_{k} \ll k^{-3 / 4}$. In fact, one sees $c_{k} k^{3 / 4}$ increase monotonically at first and then begin to oscillate around zero, where the wavelength fits well with the imaginary part of the first critical zero of $\zeta(s)$, see Figure 1.1.

\section{Elementary properties of the Pochhammer polynomials}

We begin with

$$
(-1)^{k}\left(\begin{array}{c}
\frac{s}{2}-1 \\
k
\end{array}\right)=P_{k}\left(\frac{s}{2}\right),
$$

which is essentially a matter of notation.

The proofs of the following lemmas are rather standard, but we give them here for the sake of completeness. 
The $k$ th-degree polynomial $P_{k}(s)$ grows like $s^{k}$ for large $|s|$; more precisely, we can state the following lemma.

LEMMA 2.1.

$$
\left|P_{k}(s)\right|>\frac{|s|^{k}}{k ! 2^{k}} \quad(|s|>2 k)
$$

Proof. The condition $|s|>2 k$ implies that $|1-r / s|>1 / 2$ for $r=1,2, \ldots, k$, thus

$$
\left|P_{k}(s)\right|=\prod_{r=1}^{k}\left|\frac{r-s}{r}\right|=\frac{|s|^{k}}{k !} \prod_{r=1}^{k}\left|1-\frac{r}{s}\right|>\frac{|s|^{k}}{k ! 2^{k}} .
$$

The following is the fundamental limit relation connecting the Pochhammer polynomials to the gamma function.

LEMMA 2.2. Uniformly on compact sets,

$$
\lim _{k \rightarrow \infty} P_{k}(s) k^{s}=\frac{1}{\Gamma(1-s)}
$$

Proof. From $\sum_{r=1}^{k}(1 / r)=\gamma+\log k+O(1 / k)$ and the infinite product for the gamma function, we obtain

$$
P_{k}(s) k^{s}=e^{s O(1 / k)} e^{-\gamma s} \prod_{r=1}^{k}\left(1-\frac{s}{r}\right) e^{s / r} \longrightarrow \frac{1}{-s \Gamma(-s)}=\frac{1}{\Gamma(1-s)}
$$

the convergence being uniform on compact sets of the plane.

An immediate corollary of Lemma 2.2 is the following lemma.

Lemma 2.3. For every compact set $H \subset \mathbb{C}$, there is a positive constant $C_{H}$, not depending on $k$, such that

$$
\left|P_{k}(s)\right| \leq C_{H} k^{-\Re(s)} \quad(s \in A, k=1,2, \ldots) .
$$

Proof. Write the uniform limit (2.4) as

$$
\left|P_{k}(s) k^{s}-\frac{1}{\Gamma(1-s)}\right| \leq \epsilon_{H}(k) \longrightarrow 0 \quad(s \in H, k=1,2, \ldots)
$$

therefore

$$
\left|P_{k}(s)\right| \leq\left(\frac{1}{|\Gamma(1-s)|}+\epsilon_{H}(k)\right) k^{-\mathfrak{R}(s)}
$$


LEMMA 2.4. For $\mathfrak{R}(s)<0$,

$$
\begin{gathered}
\frac{1}{\left|P_{k}(s)\right|}>\frac{1}{\left|P_{k+1}(s)\right|} \quad(k \geq 1), \\
\frac{1}{\left|P_{k}(s)\right|} \longrightarrow 0 \quad(k \longrightarrow \infty) .
\end{gathered}
$$

Proof. Lemma 2.2 clearly implies that

$$
\frac{1}{\left|P_{k}(s)\right|} \longrightarrow 0 \quad(\mathfrak{R}(s)<0)
$$

and for $\mathfrak{R}<<0$, the trivial inequality $|w| \geq|\mathfrak{R} w|$ yields

$$
\left|\frac{P_{k+1}(s)}{P_{k}(s)}\right|=\left|1-\frac{s}{k+1}\right| \geq 1-\frac{\mathfrak{R}(s)}{k+1}>1,
$$

so the sequence $1 / P_{k}(s)$ is strictly decreasing.

The next lemma establishes an interesting connection between the partial fraction decomposition of $1 / P_{k}(s)$ and the iterated forward difference operator involved in definition (1.1).

LEMMA 2.5.

$$
\frac{1}{P_{k}(s)}=\sum_{j=1}^{k}(-1)^{j}\left(\begin{array}{l}
k \\
j
\end{array}\right) \frac{j}{s-j}, \quad k \geq 1 .
$$

The proof of this lemma is an elementary exercise in computing

$$
\operatorname{Res}\left(\frac{1}{P_{k}(s)} ; s=j\right)=(-1)^{j}\left(\begin{array}{l}
k \\
j
\end{array}\right) j, \quad j=1,2, \ldots, k \text {. }
$$

\section{Proof of the main theorem (Theorem 1.1)}

3.1. Proof of sufficiency. The sufficiency of the condition (1.5) follows from writing $1 / \zeta(s)$ as a series of Pochhammer polynomials. We state it as a separate proposition as we believe it deserves special attention.

Proposition 3.1. If $c_{k} \ll k^{-3 / 4+1 / 2 \epsilon}$ for any $\epsilon>0$, then

$$
\frac{1}{\zeta(s)}=\sum_{k=0}^{\infty} c_{k} P_{k}\left(\frac{s}{2}\right), \quad \mathfrak{R}(s)>\frac{1}{2},
$$

where the series converges uniformly on compact subsets of the half-plane. A fortiori, $\zeta(s)$ does not vanish for $\mathfrak{R}(s)>1 / 2$.

Remark 3.2. Since it will be shown that actually $c_{k} \ll k^{-1 / 2}$, it follows rather trivially that the representation (3.1) for $1 / \zeta(s)$ is unconditionally valid at least in the half-plane 
$\mathfrak{R}(s)>1$. On the other hand, as announced in (1.4), Proposition 3.1 shows that

$$
c_{k} \nless k^{-3 / 4-\delta} \quad(\forall \delta>0),
$$

since the contrary statement would imply by $(3.1)$ that $\zeta(s)$ has no zeros on the critical line.

We need a lemma before proving Proposition 3.1.

Lemma 3.3. Define

$$
q_{k}:=\sum_{n=1}^{\infty} \frac{1}{n^{2}}\left(1-\frac{1}{n^{2}}\right)^{k}
$$

then

$$
q_{k} \ll k^{-1 / 2} \text {. }
$$

Proof. The contribution of the terms with $n>\sqrt{k}$ is trivially $\ll k^{-1 / 2}$, whereas the contribution of the remaining terms is

$$
\sum_{n \leq \sqrt{k}} \frac{1}{n^{2}}\left(1-\frac{1}{n^{2}}\right)^{k} \ll \int_{1}^{\infty} e^{-k / x^{2}} \frac{d x}{x^{2}}=\frac{k^{-1 / 2}}{2} \int_{0}^{\infty} e^{-u} u^{-1 / 2} d u \ll k^{-1 / 2} .
$$

Proof of Proposition 3.1. First, note that

$$
\begin{aligned}
c_{k} & =\sum_{j=0}^{k}(-1)^{j}\left(\begin{array}{l}
k \\
j
\end{array}\right) \frac{1}{\zeta(2 j+2)} \\
& =\sum_{j=0}^{k}(-1)^{j}\left(\begin{array}{l}
k \\
j
\end{array}\right) \sum_{n=1}^{\infty} \frac{\mu(n)}{n^{2 j+2}} \\
& =\sum_{n=1}^{\infty} \frac{\mu(n)}{n^{2}} \sum_{j=0}^{k}(-1)^{j}\left(\begin{array}{l}
k \\
j
\end{array}\right) \frac{1}{n^{2 j}} \\
& =\sum_{n=1}^{\infty} \frac{\mu(n)}{n^{2}}\left(1-\frac{1}{n^{2}}\right)^{k} .
\end{aligned}
$$

For $\mathfrak{R}(s)>1$, we have

$$
\begin{aligned}
\frac{1}{\zeta(s)} & =\sum_{n=1}^{\infty} \frac{\mu(n)}{n^{s}}=\sum_{n=1}^{\infty} \frac{\mu(n)}{n^{2}}\left(\frac{1}{n^{2}}\right)^{s / 2-1} \\
& =\sum_{n=1}^{\infty} \frac{\mu(n)}{n^{2}}\left(1-\left(1-\frac{1}{n^{2}}\right)\right)^{s / 2-1} \\
& =\sum_{n=1}^{\infty} \frac{\mu(n)}{n^{2}} \sum_{k=0}^{\infty}(-1)^{k}\left(\frac{s}{2}-1\right)\left(1-\frac{1}{n^{2}}\right)^{k} \\
& =\sum_{n=1}^{\infty} \frac{\mu(n)}{n^{2}} \sum_{k=0}^{\infty} P_{k}\left(\frac{s}{2}\right)\left(1-\frac{1}{n^{2}}\right)^{k} .
\end{aligned}
$$


These summations can be interchanged because letting

$$
S=\sum_{n=1}^{\infty} \sum_{k=0}^{\infty} \frac{1}{n^{2}}\left|P_{k}\left(\frac{s}{2}\right)\right|\left(1-\frac{1}{n^{2}}\right)^{k}
$$

we see from Lemmas 2.3 and 3.3 that

$$
\begin{aligned}
S & =\sum_{k=0}^{\infty}\left|P_{k}\left(\frac{s}{2}\right)\right| \sum_{n=1}^{\infty} \frac{1}{n^{2}}\left(1-\frac{1}{n^{2}}\right)^{k} \\
& =\sum_{k=0}^{\infty}\left|P_{k}\left(\frac{s}{2}\right)\right| q_{k} \ll \sum_{k=1}^{\infty} k^{-\Re(s) / 2-1 / 2}<\infty .
\end{aligned}
$$

Thus, we proceed to interchange summations in (3.7), taking into account (3.6), to obtain, unconditionally for $\mathfrak{R}(s)>1$,

$$
\frac{1}{\zeta(s)}=\sum_{k=0}^{\infty} P_{k}\left(\frac{s}{2}\right) \sum_{n=1}^{\infty} \frac{\mu(n)}{n^{2}}\left(1-\frac{1}{n^{2}}\right)^{k}=\sum_{k=0}^{\infty} c_{k} P_{k}\left(\frac{s}{2}\right) .
$$

But Lemma 2.3, together with the hypothesis $c_{k} \ll k^{-3 / 4+(1 / 2) \epsilon}$, implies that the above series converges uniformly on compacts of the half-plane $\mathfrak{R}(s)>1 / 2+\epsilon$. Thus, the series extends $1 / \zeta(s)$ analytically to the half-plane $\mathfrak{R}(s)>1 / 2$. We have thus proved the validity of (3.1).

Finally, we prove the assertion on simple zeros in the main theorem (Theorem 1.1). Assume that $c_{k} \ll k^{-3 / 4}$. Take any fixed $s=1 / 2+i \beta$ on the critical line and $0<h \leq \delta$ for a fixed, finite $\delta>0$. By (3.1),

$$
\left|\frac{1}{\zeta(s+h)}\right| \leq\left|c_{0}\right|+\sum_{k=1}^{\infty} O\left(k^{-3 / 4}\right)\left|P_{k}\left(\frac{s+h}{2}\right)\right| .
$$

Now it is clear that

$$
\alpha_{1}=\sup _{0 \leq h \leq \delta} \frac{1}{|\Gamma(3 / 4+h / 2-i(\beta / 2))|}<\infty
$$

But by Lemma 2.2, there is a constant $\alpha_{2}>0$ such that

$$
\sum_{k=1}^{\infty} k^{-3 / 4}\left|P_{k}\left(\frac{s+h}{2}\right)\right| \leq \alpha_{2} \sum_{k=1}^{\infty} \frac{k^{-1-h / 2}}{|\Gamma(3 / 4+h / 2-i(\beta / 2))|} \leq \alpha_{1} \alpha_{2} \zeta\left(1+\frac{h}{2}\right) \ll \frac{1}{h} .
$$

Applying this in (3.11), we obtain

$$
\left|\frac{1}{\zeta(s+h)}\right| \ll \frac{1}{h}
$$

This shows that if $\zeta(s)=0$, then $s$ can only be a simple zero. 


\subsection{Necessity of the condition}

Proof of the necessity of the condition. Assume now that the Riemann hypothesis is true. If, as usual, we write

$$
M(x):=\sum_{n \leq x} \mu(n)
$$

we then have

$$
M(x) \ll x^{1 / 2+2 \epsilon} \quad(\forall \epsilon>0)
$$

which, actually, is well known to be equivalent to the Riemann hypothesis (see, e.g., [8, Theorem 14.25(C)]).

We can transform the second expression for $c_{k}$ in (3.6) summing it by parts to obtain

$$
\begin{aligned}
c_{k} & =-\int_{1}^{\infty} M(x) \frac{d}{d x}\left(\frac{1}{x^{2}}\left(1-\frac{1}{x^{2}}\right)^{k}\right) d x \\
& =2 \int_{0}^{1} M\left(\frac{1}{x}\right)\left(1-x^{2}\right)^{k-1}\left(x-(k+1) x^{3}\right) d x .
\end{aligned}
$$

Therefore,

$$
\left|c_{k}\right| \leq 2(k+1) \int_{0}^{1}\left|M\left(\frac{1}{x}\right)\right| x^{3}\left(1-x^{2}\right)^{k-1} d x+2 \int_{0}^{1}\left|M\left(\frac{1}{x}\right)\right| x\left(1-x^{2}\right)^{k-1} k d x,
$$

but (on the Riemann hypothesis)

$$
M\left(\frac{1}{x}\right) \ll x^{-1 / 2-2 \epsilon} \quad(x \downarrow 0),
$$

so that

$$
c_{k} \ll k \int_{0}^{1} x^{5 / 2-2 \epsilon}\left(1-x^{2}\right)^{k-1} d x+\int_{0}^{1} x^{1 / 2-2 \epsilon}\left(1-x^{2}\right)^{k-1} d x .
$$

On the other hand, for $\mathfrak{R}(\lambda)>-1$, a classical beta integral result (see, e.g., [2, Section 9.3]) gives

$$
\int_{0}^{1} x^{\lambda}\left(1-x^{2}\right)^{k-1} d x=\frac{1}{2} \Gamma\left(\frac{1}{2}(\lambda+1)\right) \frac{\Gamma(k)}{\Gamma(k+(1 / 2)(\lambda+1))} \ll k^{-1 / 2-\lambda / 2},
$$

where the last estimate follows from Stirling's formula for the logarithm of the gamma function; hence (3.20) becomes

$$
c_{k} \ll k^{-3 / 4+\epsilon}
$$




\section{An exact formula for $c_{k}$}

The $c_{k}$ have a nice exact expression as an integral in the complex plane, as shown by the following proposition.

Proposition 4.1.

$$
-2 k c_{k-1}=\frac{1}{2 \pi i} \int_{a-i \infty}^{a+i \infty} \frac{d s}{\zeta(s) P_{k}(s / 2)} \quad(k \geq 2,1<a<2),
$$

where the integral is absolutely convergent. The path of integration is the line $\mathfrak{R}(s)=a$ traversed in the upward direction.

Proof. Note first that for any $\sigma>1$,

$$
\frac{1}{|\zeta(s)|}=\left|\sum_{n=1}^{\infty} \frac{\mu(n)}{n^{s}}\right| \leq \sum_{n=1}^{\infty} \frac{1}{n^{\sigma}}=\zeta(\sigma)
$$

By Lemma 2.1, we may move the path of integration to a vertical line with any abscissa $b>2 k$. Calculating the residues with the help of Lemma 2.5, we get

$$
\frac{1}{2 \pi i} \int_{a-i \infty}^{a+i \infty} \frac{d s}{\zeta(s) P_{k}(s / 2)}=\sum_{j=1}^{k}(-1)^{j}\left(\begin{array}{c}
k \\
j
\end{array}\right) \frac{2 j}{\zeta(2 j)}+\frac{1}{2 \pi i} \int_{b-i \infty}^{b+i \infty} \frac{d s}{\zeta(s) P_{k}(s / 2)}
$$

but

$$
-2 k \sum_{j=0}^{k-1}(-1)^{j}\left(\begin{array}{c}
k-1 \\
j
\end{array}\right) \frac{1}{\zeta(2 j+2)}=-2 k c_{k-1} .
$$

For fixed $k$, let $b \rightarrow+\infty$. By Lemma 2.1, this yields

$$
\frac{1}{2 \pi i} \int_{b-i \infty}^{b+i \infty} \frac{d s}{\zeta(s) P_{k}(s / 2)} \longrightarrow 0 \quad(b \longrightarrow \infty)
$$

so that (4.1) follows.

Proof of the explicit formula (Theorem 1.5). We intend now to move the path of integration in (4.1) to the left of the critical strip. As this procedure is a little more delicate than that of the previous lemma, we will proceed in more detail. We begin with a fixed but arbitrary $k \geq \max (4, A)$, for some fixed $A>0$ to be determined later in the proof, so that, of course, to begin with Proposition 4.1 can be applied. For any $T_{v}>0$, consider the integral

$$
I(k, \nu):=\frac{1}{2 \pi i} \int_{L_{\nu}} \frac{d s}{\zeta(s) P_{k}(s / 2)},
$$

where $L_{v}$ is the rectangle $\left\{3 / 2-i T_{\nu}, 3 / 2+i T_{\nu},-1+i T_{\nu},-1-i T_{\nu}\right\}$ traversed in the positive direction. By the residue theorem, we have

$$
I(k, v)=\sum_{|\mathfrak{I} \rho|<T_{\nu}} \operatorname{Res}\left(\frac{1}{\zeta(s) P_{k}(s / 2)} ; s=\rho\right),
$$


where the finite sum runs over the zeros $\rho$ of the zeta function in the interior of the rectangle $L_{v}$. The choice of the $T_{\nu}$ is dictated by Theorem 9.7 in Titchmarsh's monograph [8], where it is attributed to Valiron et al., independently. As a consequence of this unconditional theorem for some constant $A>0$, there is a sequence $T_{\nu}$ with $\nu<T_{\nu}<v+1$ such that

$$
\frac{1}{\left|\zeta\left(\sigma+i T_{v}\right)\right|}<T_{\nu}^{A} \quad(-1 \leq \sigma \leq 2) .
$$

This estimate, together with Lemma 2.1, implies that the contribution of the horizontal rungs in $I(k, v)$ tends to zero as $v \rightarrow \infty$.

On the other hand, it is clear that as $\nu \rightarrow \infty$, the integral on the right-hand vertical side of $L_{\nu}$ tends to the absolutely convergent integral on the right-hand side of (4.1), thus to $-2 k c_{k-1}$.

Likewise, to see that the contribution of the left-hand side of the rectangle $L_{\gamma}$ converges as $v \rightarrow \infty$ to

$$
J_{k}:=-\frac{1}{2 \pi i} \int_{-1-i \infty}^{-1+i \infty} \frac{d s}{\zeta(s) P_{k}(s / 2)},
$$

it suffices to show that this integral is absolutely convergent. To prove this, note that the functional align implies that

$$
\begin{aligned}
\frac{1}{|\zeta(-1+i t)|} & =\frac{1}{\left|2^{1-i t} \pi^{-2-i t} \cos (i \pi t / 2) \Gamma(2-i t) \zeta(2-i t)\right|} \\
& \ll \frac{1}{e^{\pi t / 2}+e^{-\pi t / 2}} \frac{1}{|\Gamma(2-i t)|} \ll|t|^{3 / 2},
\end{aligned}
$$

where we used again (4.2) in writing $\left|\zeta(2-i t)^{-1}\right| \leq \zeta(2)$, and the well-known estimates for the gamma function on vertical strips (see, e.g., formula (21.52) in Rademacher's treatise [6]). Now (4.10) and the trivial bound (2.2) yield the absolute integrability of (4.9).

We have thus proved that the limit as $v \rightarrow \infty$ of $I(k, v)$ exists, arriving at

$$
-2 k c_{k-1}=\lim _{\nu \rightarrow \infty} \sum_{|\mathfrak{I} \rho|<T_{\nu}} \operatorname{Res}\left(\frac{1}{\zeta(s) P_{k}(s / 2)} ; s=\rho\right)+J_{k},
$$

where the limit of the summation has also been shown to exist. But $J_{k} \rightarrow 0$ as $k \rightarrow \infty$ by the monotone convergence theorem on account of Lemma 2.4. This completes the proof of (1.11) of Theorem 1.5, which immediately implies (1.12) under the assumption of simple zeros.

\section{References}

[1] L. Báez-Duarte, On Maslanka's representation for the Riemann zeta function, preprint, 2003, available at: http://arxiv.org/abs/math.NT/0307214v.1.

[2] E. T. Copson, An Introduction to the Theory of Functions of a Complex Variable, Clrendon Press, Oxford, 1935, reprinted 1966. 
[3] G. H. Hardy and J. E. Littlewood, Contributions to the theory of the Riemann zeta-function and the theory of the distribution of primes, Acta Math. 41 (1918), 119-196.

[4] K. Maslanka, A hypergeometric-like representation of zeta-function of Riemann, 1997, Cracow Observatory preprint no. 1997/60.

[5] _ A hypergeometric-like representation of zeta-function of Riemann, 2001, posted at: http://arxiv.org/abs/math-ph/0105007v1.

[6] H. Rademacher, Topics in Analytic Number Theory, Die Grundlehren der mathematischen Wissenschaften, vol. 169, Springer, New York, 1973.

[7] M. Riesz, Sur l'hypothèse de Riemann, Acta Math. 40 (1916), 185-190 (French).

[8] E. C. Titchmarsh, The Theory of the Riemann Zeta-Function, Clarendon Press, Oxford, 1951.

Luis Báez-Duarte: Departamento de Matemáticas, Instituto Venezolano de Investigaciones Científicas, Apartado Postal 21827, Caracas 1020-A, Venezuela

E-mail address: lbaezd@cantv.net 


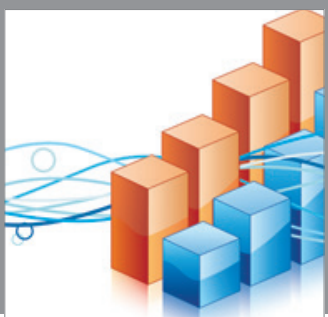

Advances in

Operations Research

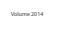

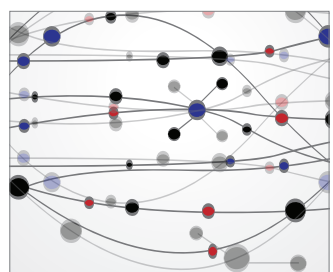

\section{The Scientific} World Journal
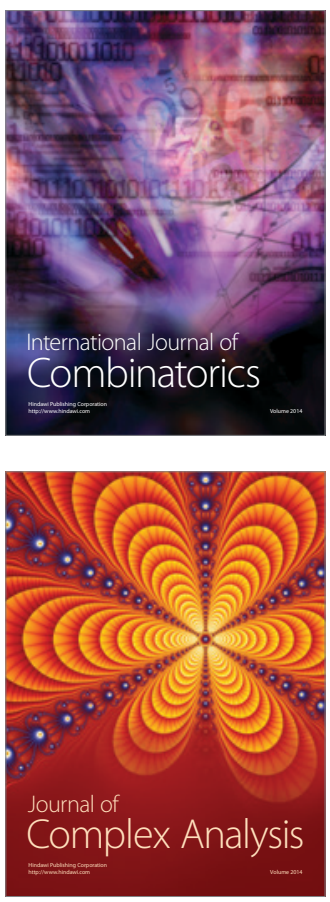

International Journal of

Mathematics and

Mathematical

Sciences
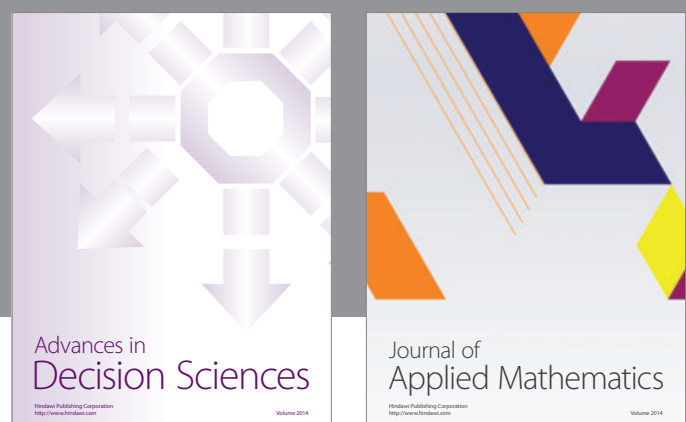

Journal of

Applied Mathematics
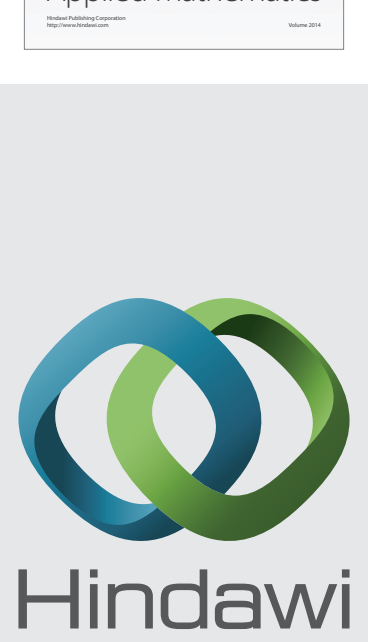

Submit your manuscripts at http://www.hindawi.com
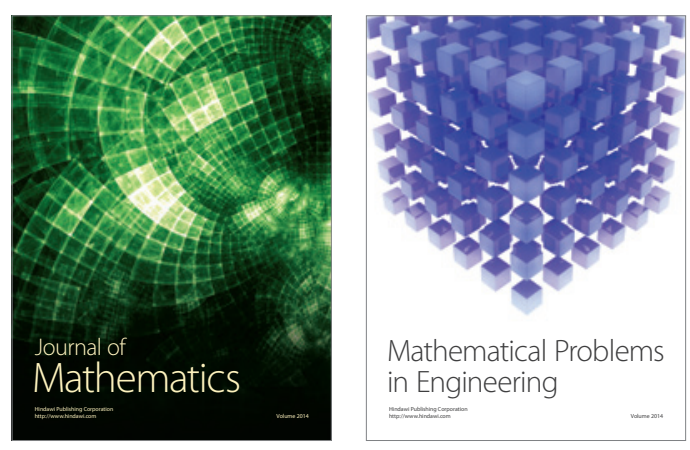

Mathematical Problems in Engineering
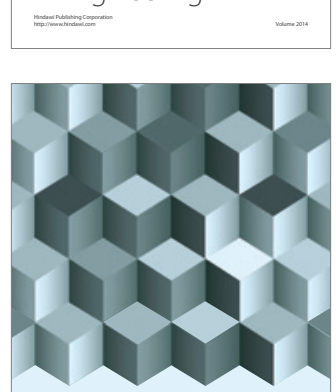

Journal of

Function Spaces
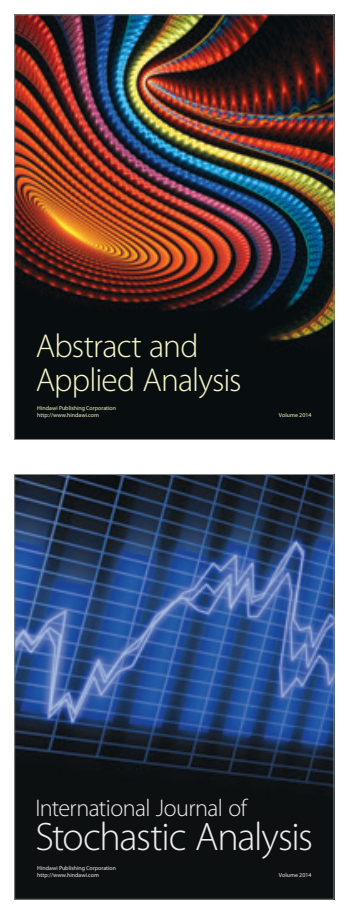

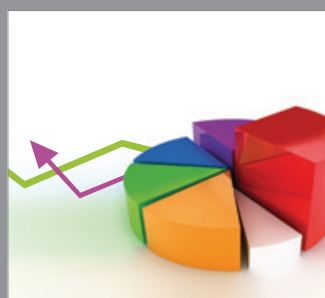

ournal of

Probability and Statistics

Promensencen
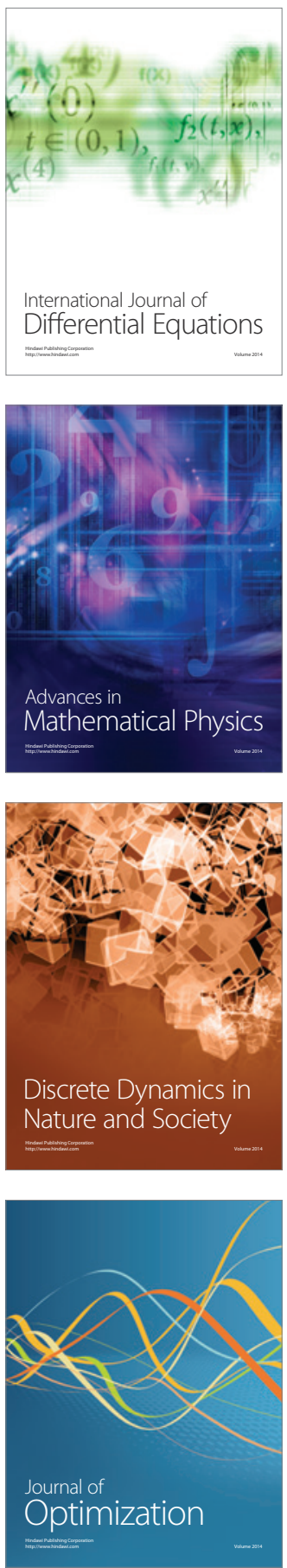